\title{
LSSR1 facilitates seed setting rate by promoting fertilization in rice
}

\author{
Xiaojiao Xiang ${ }^{1,2}$, Peipei Zhang ${ }^{1}$, Ping Yu', Yingxin Zhang ${ }^{1}$, Zhengfu Yang ${ }^{1,2}$, Lianping Sun ${ }^{1}$, Weixun Wu',
} Riaz Muhammad Khan ${ }^{1}$, Adil Abbas', Shihua Cheng ${ }^{1 *}$ and Liyong Cao ${ }^{1 *}$

\begin{abstract}
Seed setting rate is one of the major components that determine rice (Oryza sativa L.) yield. Successful fertilization is necessary for normal seed setting. However, little is known about the molecular mechanisms governing this process. In this study, we report a novel rice gene, LOW SEED SETTING RATE1 (LSSR1), which regulates the seed setting rate by facilitating rice fertilization. LSSR1 encodes a putative GH5 cellulase, which is highly conserved in plants. LSSR1 is predominantly expressed in anthers during the microsporogenesis stage, and its encoded protein contains a signal peptide at the N-terminal, which may be a secretory protein that stores in pollen grains and functions during rice fertilization. To explore the physiological function of LSSR1 in rice, loss-of-function mutants of LSSR1 were created through the CRISPR-Cas9 system, which showed a significant decrease in rice seed setting rate. However, the morphology of the vegetative and reproductive organs appears normal in Issri mutant lines. In addition, Issr1 pollen grains could be normally stained by $\mathrm{I}_{2}-\mathrm{Kl}$ solution. Cytological results demonstrate that the blockage of fertilization mostly accounted for the low seed setting rate in Issr1 mutant lines, which was most likely caused by abnormal pollen grain germination, failed pollen tube penetration, and retarded pollen tube elongation. Together, our results suggest that LSSR1 plays an important role in rice fertilization, which in turn is vital for maintaining rice seed setting rate.
\end{abstract}

Keywords: Cellulase, Microsporogenesis, Secretory protein, CRISPR-Cas9 system, Spikelet sterility

\section{Background}

Rice (Oryza sativa L.), the staple food of more than half of the world's population particularly distributed in Africa and Asia, is critical for global food security (Seck et al. 2012). Panicle number, grain number per panicle, grain weight, and seed setting rate are the major components that determine rice yield. In the past few decades, excellent progress has been made in illustrating the molecular mechanisms of panicle number ( $\mathrm{Li}$ et al. 2003; Takeda et al. 2003; Xu et al. 2012; Wang et al. 2017), grain number per panicle (Ashikari et al. 2005; Wu et al. 2016; Huo et al. 2017), and grain weight ( $\mathrm{Li}$ et al. 2011; Ishimaru et al. 2013; Duan et al. 2017). Although recent results have shed some light on the molecular regulation of the seed setting rate in rice (Zhou et al. 2011; Li et al.

\footnotetext{
* Correspondence: chengshihua@caas.cn; caoliyong1966@163.com

${ }^{1}$ Key Laboratory for Zhejiang Super Rice Research and State Key Laboratory of Rice Biology, China National Rice Research Institute, Hangzhou 310006, China

Full list of author information is available at the end of the article
}

2013; Zhu et al. 2017), much remains unknown. Decreased seed setting rate has become a bottleneck that limits further improvement in rice grain yield using hybrid rice (Dan et al. 2014; Li et al. 2016). Additionally, climate variation may result in huge losses of rice yield, since the seed setting rate is susceptible to environmental conditions. Therefore, identifying more genes that control rice seed setting rate and detailing their genetic mechanisms, as well as their application in rice product is of critical importance for the maintenance and improvement of rice yield.

Many factors can lead to spikelet sterility. These include defected pollen grains, abnormal embryo sacs, inappropriate temperature at the reproductive stage, etc. All of these could in turn result in a low rice seed-setting-rate. Successful double fertilization is the first guarantee for spikelet fertility. The process of fertilization in angiosperms goes as follows. Pollen grains land on the stigma, hydrate, and germinate to form a specialized structure called a pollen tube through 
cell-cell interaction with the pistil (Yadegari and Drews 2004; Palanivelu and Johnson 2010). Pollen tubes then penetrate the stigma and style and reach the transmitting tract (TT) (Jiang 2005). Next, pollen tubes elongate through the TT under the guidance of the pistil to reach the ovules (Márton and Dresselhaus 2010). Finally, the pollen tubes traverse the micropyle and synergid cells, rupture, and release the two sperms to finish double fertilization (Sandaklie-Nikolova et al. 2007; Leydon et al. 2015; Ge et al. 2017). PSS1 is a kinesin-1 family gene, which plays an important role in regulating the seed setting rate of rice by controlling male meiotic chromosomal dynamics, male gametogenesis, and anther dehiscence (Zhou et al. 2011). Rice PTB1, encoding a RING-type E3 ubiquitin ligase, positively regulates the seed setting rate by facilitating pollen tube growth and is affected by the environmental temperature ( $\mathrm{Li}$ et al. 2013). OsCNGC13, a cyclic nucleotide-gated channel protein, acts as a novel maternal sporophytic factor required for stylar $\left[\mathrm{Ca}^{2+}\right]_{\mathrm{cyt}}$ (cytoplasmic calcium concentration) accumulation, ECM (extracellular matrix) component modification, and STT (style transmission tissue) cell death, thus promoting the pollen tube elongation and seed setting in rice (Zhu et al. 2017). Nevertheless, little is known about the correlation between rice seed setting rate and other steps.

During the step in which the pollen tubes penetrate the stigma, they must first penetrate the cuticle and cell wall to enter the papillar cell (Jiang 2005). Wall hydrolases and wall-modulating proteins are known either to be present, derived from the tapetum cells, or to be temporarily secreted in the pollen grains (Suen et al. 2003). They could be specifically synthesized for hydrolysis of the stigma wall, or the tube track wall for pollen tube growth (Suen and Huang 2006). Alternatively, they might control the structure and strength of the expanding pollen tube tips (Bosch and Hepler 2005). The rice cell wall is a type II cell wall, which consists of cellulose, arabinoxylans, glucuronoarabinoxylan, and so on (Carpita and Gibeaut 1993). Glycosyl hydrolases (GHs) in rice pollens during pollen tube growth may act on these polysaccharides in the stigma wall, facilitating the entry of the pollen tubes into the pistil. GHs can be grouped into "clans" according to their three-dimensional (3D) structures (Henrissat and Davies 1997). Clan A is the largest group, which contains a core $(\beta / \alpha)_{8}$ architecture with an acid/base and a nucleophile on the ends of $\beta$-strands 4 and 7 (Jenkins et al. 1995). Glycosyl hydrolase family 5 (GH5) is one of the clan A families that contains enzymes with a wide range of catalytic activities, including cellulases, endo- $\beta-1,4$-xylanase, $\beta$-mannosidase, chitosanase, $\beta$-primeverosidase, etc... In maize (Zea mays $L$.), the GH5 xylanase $Z m X Y N 1$ has been proved to facilitate the penetration of pollen tube into silk during sexual reproduction (Suen and Huang 2006). GH5BG is a GH5 $\beta$-glucosidase containing a fascin-like domain, which can be up-regulated in response to stress in rice seedlings (Opassiri et al. 2007). However, no study on the physiological role of rice GH5 cellulases has ever been reported.

Here we report a new rice gene, LOW SEED SETTING RATE1 (LSSR1), which is predicted to encode a GH5 cellulase and regulates the seed setting rate of rice. LSSR1 is predominantly expressed in the rice anther from the premiotic stage to the single-cell pollen stage. Loss-of-function mutation of LSSR1 achieved through the CRISPR-Cas9 system has no effect on the morphology of the vegetative and reproductive organs, as well as the pollen $\mathrm{I}_{2}-\mathrm{KI}$ staining in rice. Nonetheless, the mutation in LSSR1 resulted in either a decreased number or none of the pollen tubes reaching to the ovule during fertilization, leading ultimately to a low seed setting rate. The results suggest that LSSR1 plays a critical role in the process of rice fertilization.

\section{Materials and methods Materials and growth conditions}

All materials in this study were with a Nipponbare (Oryza sativa Japonica) background. Nipponbare (WT) and lssr 1 mutant lines were grown in experimental fields at the China National Rice Research Institute in Hainan or Hangzhou during the normal growing seasons.

\section{RNA isolation, CDNA synthesis, and qRT-PCR}

Total RNA was extracted from various rice tissues using the RNAprep Pure Plant Kit (Tiangen, China) according to the manufacturer's protocol. For qRT-PCR analysis, isolated total RNA was first adjusted to the same concentration and then reverse-transcribed with the ReverTra Ace qPCR RT Master Mix with gDNA Remover (Toyobo, Japan). qRT-PCR was performed with a LightCycler 480 (Roche, Germany) using LightCycler ${ }^{\circ} 480$ SYBR $^{\circ}$ Green I Master Mix (Roche, USA) in accordance with the manufacturer's instructions. Measurements were obtained using the relative quantification method (Livak and Schmittgen 2001). The rice gene OsActin (LOC_Os03g50885; http:// rice.plantbiology.msu.edu/) was used as the internal control (Zhang et al. 2017). Each reaction was performed with three replicates. Primers used for qRT-PCR are listed in Additional file 1: Table S1.

\section{Phylogenetic analysis}

The protein sequence of LSSR1 (LOC_OsO2g38260) was used as a BLAST query to identify putative homologs on the NCBI database. Protein sequence alignments were made using ClustalW with default parameters and analyzed by GENEDOC. The alignment was used to construct a maximum likelihood phylogenetic tree with 500 bootstrap replications, a Poisson model, and other default parameters. The signal sequence was predicted with the TargetP 1.1 Server (http://www.cbs.dtu.dk/services/ 
TargetP/). The structure, molecular model, and active sites of LSSR1 were predicted using Phyre2 (http://www. sbg.bio.ic.ac.uk/phyre $2 / \mathrm{html} /$ page.cgi?id=index) by comparing with Pyrococcus horikoshii endocellulase c2zunB (Wass et al. 2010; Kim and Ishikawa 2011; Kelley et al. 2015). The three-dimensional structure was analyzed by software VMD.

\section{Subcellular localization of LSSR1 through transient expression in $N$. benthamiana}

The full-length CDS of LSSR1 was amplified from the wild-type cDNA and fused with the 5 ' terminus and 3' terminus of the coding sequence of EGFP (BamHI-HindIII sites) in pYBA 1132 and pYBA 1152 vectors, respectively (Liu et al. 2017). The recombinant proteins were transiently expressed or co-expressed with mCherry alone in $N$. benthamiana leaves. Confocal imaging analysis was performed using a laser scanning confocal microscope (ZEISS LSM 750, Germany) 3-5 days after infiltration. Primers used for the generation of these fusion constructs are listed in Additional file 1: Table S1.

\section{Construction of Issr1}

The lssr1 mutants were generated using the CRISPR/ Cas9 system according to the methods of previous studies (Miao et al. 2013; Huang et al. 2017). Three CRISPR targets for LSSR1 were selected using CRISPR-P v2.0 (http://cbi.hzau.edu.cn/CRISPR2/). The spacers were cloned by ligating complementary oligos into a type II restriction site $(B s a \mathrm{I})$. Gateway recombination was used to incorporate guide sgRNA into vector pBWA(V)H_cas9i2, which contains Cas9 driven by P35S for expression. The recombinant vectors were then transferred into the Nipponbare callus for the culture of transgenic lines. The genomic DNA of transgenic lines was extracted using the modified CTAB protocol (Murray and Thompson 1980). The genomic region surrounding the CRISPR target site for LSSR1 was amplified and sequenced to screen for mutants. Mutation identification was done using SeqMan or DSDecode (http://dsdecode. scgene.com/). For mutants with a mutation in the first CRISPR target, homozygous $\mathrm{T}_{3}$ plants without T-DNA were used for phenotyping. The transgene-free mutant individuals were identified using the CRISPR/Cas9 vector specific primer. All primers used for sequencing and T-DNA detection are listed in Additional file 1: Table S1.

\section{Characterization of the mutant phenotype}

The plant morphology of the WT and lssr 1 mutant lines was photographed using a digital camera (Nikon HB-40, Japan). Florets and mature panicles were scanned on a scanner (Epson Perfection v330 Photo, Indonesia). Pistils, stamens, and $\mathrm{I}_{2}-\mathrm{KI}$ stained pollen grains were imaged using a microscope (LEICA DM4 B, Germany). $\mathrm{I}_{2}$-KI staining was used to primarily evaluate pollen viability with $\mathrm{I}_{2}$-KI staining buffer that contained $1 \%(w / v) \mathrm{I}_{2}$ and $8 \%(\mathrm{w} /$ v) KI. FAA (containing an 18:1:1 (by vol.) mixture of $70 \%$ ethanol, formalin and acetic acid)-fixed and $70 \%$ ethanol-stored panicles at different developmental stages were used for the morphologic image of 3-4, 4-5, and 5-6 $\mathrm{mm}$ florets. To examine the seed setting rate of the homozygous $\mathrm{T}_{3}$ lines $\mathrm{L} 1-1 \sim \mathrm{L} 1-5$, at least 6 morphology-similar individuals per line with 5 main panicles per plant were used. For the $\mathrm{T}_{0}$ individuals with mutation in the 2 and 3 target sites, the transgenic individual without mutation in the target sites was used as the control, as its growth period showed the same with those mutants.

For scanning electron microscopy (SEM) observation, mature anthers of the WT and lssr 1 mutants were pre-fixed in $0.1 \mathrm{M}$ sodium phosphate buffer containing $2.5 \%$ glutaraldehyde $(\mathrm{pH}=7.0)$ overnight at $4{ }^{\circ} \mathrm{C}$, then rinsed thrice using $0.1 \mathrm{M}$ phosphate buffer $(\mathrm{pH}=7.0)$. The rinsed samples were post-fixed for $1.5 \mathrm{~h}$ in $2 \% \mathrm{OsO}_{4}$ (in $\mathrm{PBS}, \mathrm{pH}=7.2$ ) and rinsed thrice again, following gradient ethanol dehydration. The dehydrated samples were processed for critical point drying using a critical point dryer (Hitachi Model HCP-2, Japan), and gold coated by an ion sputter (Hitachi Model E-1010, Japan). The gold-coated samples were observed using a scanning electron microscope (Hitachi Model SU-8010, Japan).

Co-segregation analysis between the phenotype with the genotype in the segregated $\mathrm{T}_{2}$ population of L1-2 and L1-3 was conducted to confirm that it was the mutation of LSSR1 that resulted in low seed setting rates in lssr1 mutant lines. For genotyping, a CAPS (Cleaved polymorphic amplified sequence) primer $38260 \mathrm{~s} 1-\mathrm{F} / \mathrm{R}$ was used for amplification and the restriction enzyme BseNI was used for digestion (Additional file 1: Table $\mathrm{S} 1)$. The digested products were separated on a $1 \%$ agarose gel. To evaluate that the low seed setting rate in $l s s r 1$ lines resulted from their impaired male or female gametophytes, reciprocal crosses between the WT and lssr 1 lines were conducted by artificial emasculation and hand pollination for three consecutive days.

\section{Aniline blue staining of pollinated pistils}

Aniline blue staining was conducted based on previous research with some modification (Zhang et al. 2018). More than 30 pollinated pistils of the WT and lssr 1 lines were collected and fixed in the FAA fixing reagent. The fixed samples were then rinsed with distilled water and incubated in $10 \mathrm{~mol} \cdot \mathrm{L}^{-1} \mathrm{NaOH}$ for $7 \mathrm{~min}$ at $56^{\circ} \mathrm{C}$. Then the softened samples were rinsed with distilled water and stained in a $0.1 \%(w / v)$ aniline blue solution for $12 \mathrm{~h}$. Finally, the samples were examined using a fluorescence microscope (LEICA DM4 B, Germany) with DAPI channel. During the flowering time, the floret was designated 
to be flowering as long as its glumes open, and the flowered floret was marked for the following experiment.

\section{Results}

LSSR1 is predominantly expressed in anthers during the stage of microsporogenesis

A previous microarray analysis suggested that $L O W$ SEED SETTING RATE 1 (LSSR1; LOC_Os02g38260) is predominantly expressed in meiotic and single-cell pollen stage anthers (Deveshwar et al. 2011), while the data on the Rice eFP Browser (http://www.bar.utoronto. $\mathrm{ca} /$ efprice/cgi-bin/efpWeb.cgi) shows that the expression of LSSR1 in meiotic panicles is not dominant (Additional file 1: Figure S1).

To confirm the expression pattern of LSSR1, qRT-PCR was conducted using total RNA extracted from different rice tissues. Traces of LSSR1 transcripts were detected in rice stems, sheaths, florets turning to green (FWTG), florets with mature reproductive organs (FM), and florets at 5-10 min after flowering (FAF) (Fig. 1a). Slightly more
LSSR1 transcripts were detected in 5-6 $\mathrm{mm}$ florets and 6$7 \mathrm{~mm}$ florets (Fig. 1a). Dominant expression of LSSR1 was detected in 3-4 mm florets and 4-5 mm florets, which were at the premeiotic and meiotic stages, respectively (Fig. 1a, c-e). To more precisely determine these spatial and temporal patterns, LSSR1 transcripts were examined in separated anthers or pistils from the florets at different developmental stages. The most prominent expression of LSSR1 was detected in anthers from the $5-6 \mathrm{~mm}$ florets, which were at the single-celled pollen stage (Fig. 1 b, c, f). Secondary expression of LSSR1 was detected in anthers from the $3-4 \mathrm{~mm}$ florets and $4-5 \mathrm{~mm}$ florets, which were at the premeiotic and meiotic stages, respectively (Fig. 1b, c-e). Although slightly high expressions of LSSR1 were found in pistils from $<3 \mathrm{~mm}$ florets and $4-5 \mathrm{~mm}$ florets, respectively, they were greatly lower than that in the microsporogenesis-stage anthers (Fig. 1b). These results indicate that LSSR1 is mainly expressed in the anthers during microsporogenesis, including the premeiosis, meiosis, and single-cell pollen stages.

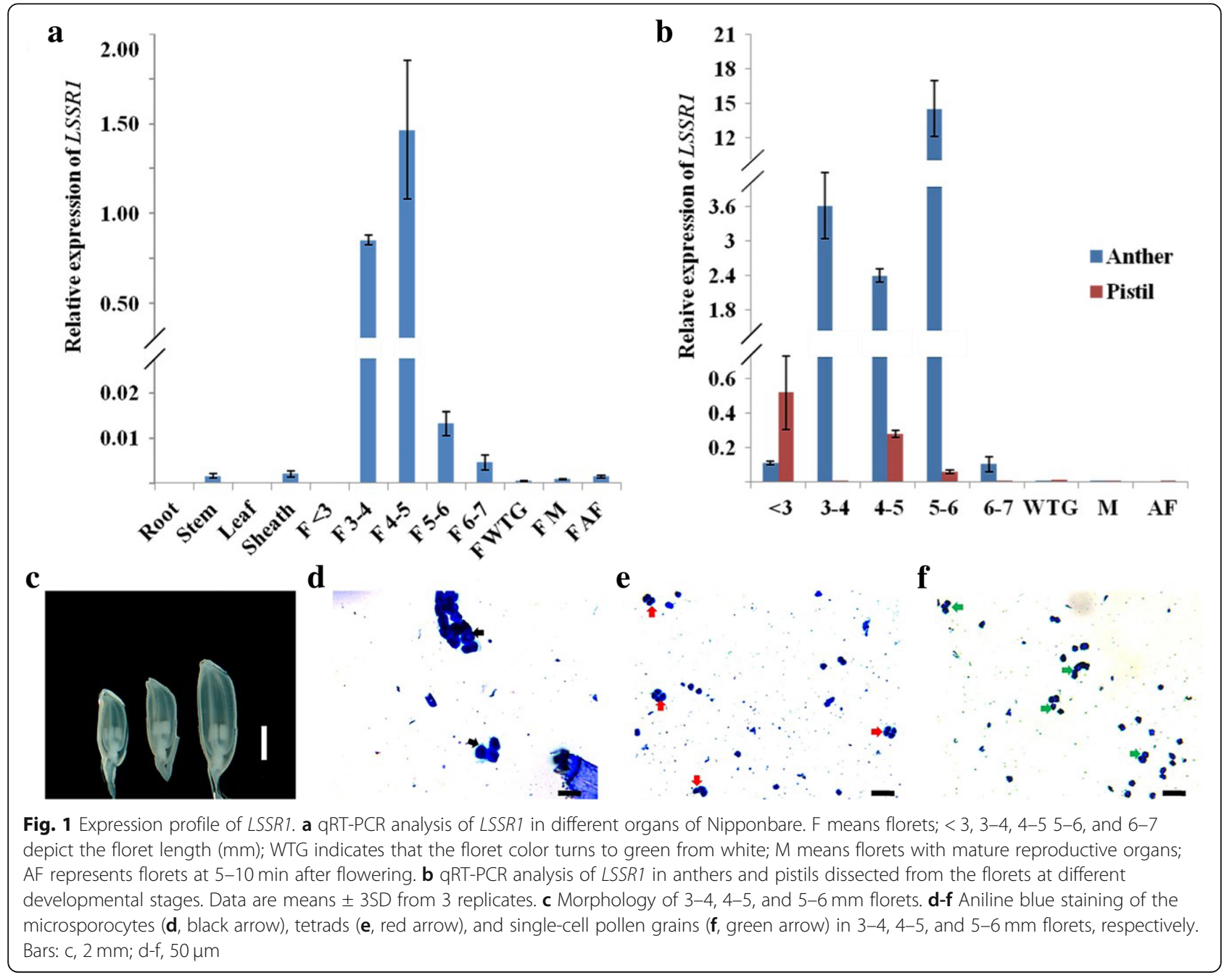


Gene cloning and sequence analysis of LSSR1

LSSR1 encodes a putative GH5 cellulase of 582 amino acids. To identify the genomic structure of LSSR1, the genomic sequence and the full length CDS were compared. The result showed that the sequence consists in two exons and one intron (Fig. 2a), which is consistent with the information on the Rice Genome Annotation Project database (http://rice.plantbiology.msu.edu/).

To examine the phylogenetic relationship between LSSR1 and its close homologs, BLASTP from the NCBI was used with the LSSR1 sequence as a query. With the threshold of identity (\%) $>50 \%$ and non-presumptive proteins in the description, three paralogs of LSSR1 in rice (LOC_Os04g40490, LOC_Os04g40500 and LOC_ Os04g40510) were found, whose encoding genes are adjacent on chromosome 4 (Fig. 2b). Coincidentally, these paralogs and LSSR1 are recorded as the four GH5 cellulases of rice in the Carbohydrate-Active enZYmes Database (CAZy) (http://www.cazy.org/GH5.html). Distinctly from LSSR1, the gene encoding the three paralogs is predicted to be expressed predominantly in rice seedling roots (LOC_Os04g40490 and LOC_Os04g40500) or young leaves (LOC_Os04g40510) (Data from transcriptomic database Rice eFP Browser). Five orthologs of LSSR1 in other plants were found, including one in Arabidopsis thaliana (GenBank, NP 172772.2) and one in Zea mays (GenBank, AQK53393.1). The ortholog in Zea mays had the closest relationship with LSSR1 (Fig. 2b). The protein sequence of LSSR1 and its homologs are highly conserved. All of them share the two putative function-conserved glutamines (an acid/base and a nucleophile on $\beta$-strands 4 and 7, respectively) as GH5 hydrolases (Fig. 3). LSSR1 is predicted to have 12 active sites, including 4 catalytic sites, 7 binding sites and 1 residue having both activities, when considering the archaeon Pyrococcus horikoshii cellulase c2zunB as a template (Fig. 3). In addition, most of the predicted active sites are conserved in LSSR1 and its homologs (Fig. 3). Morever, LSSR1 contains a signal peptide at the $\mathrm{N}$-terminal, which is predicted as a secretory protein (Fig. 3).

\section{LSSR1 is localized to the nucleus and cytoplasm through transient expression}

To evaluate whether LSSR1 is localized to the apoplast or other cell components, transient expression of the full-length CDS of LSSR1 fused with EGFP in $N$. benthamiana leaves was conducted. Unexpectedly, the fluorescence signals of both LSSR1-EGFP and EGFPLSSR1 proteins were observed in the nucleus and cytoplasm, which was the same localization of the EGFP protein alone control (Fig. 4a). To confirm this result, LSSR1-EGFP was co-expressed with mCherry alone in $N$. benthamiana leaves. As a result, LSSR1-EGFP could mainly be co-localized with mCherry (Fig. 4b). Since EGFP is pH sensitive, it may not fluoresce in the acidic environments such as the apoplast. Therfore, mCherryLSSR1 and LSSR1-mCherry fused protein were transiently co-expressed with EGFP alone in the N. benthamiana leaves. The fluorescence of both mCherry-LSSR1 and LSSR1-mCherry could also be observed in the nucleus and cytosol, and could mainly be co-localized with

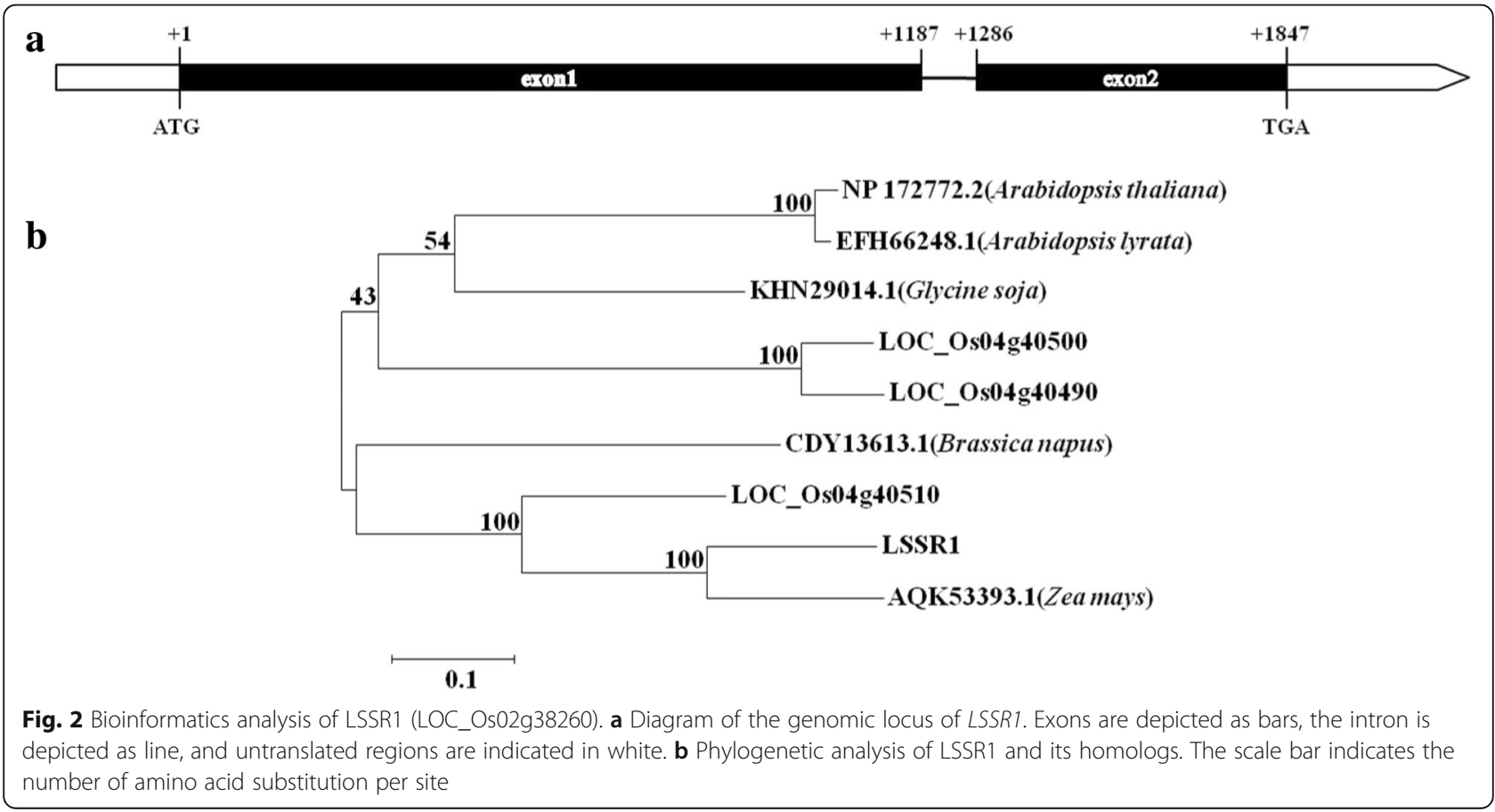




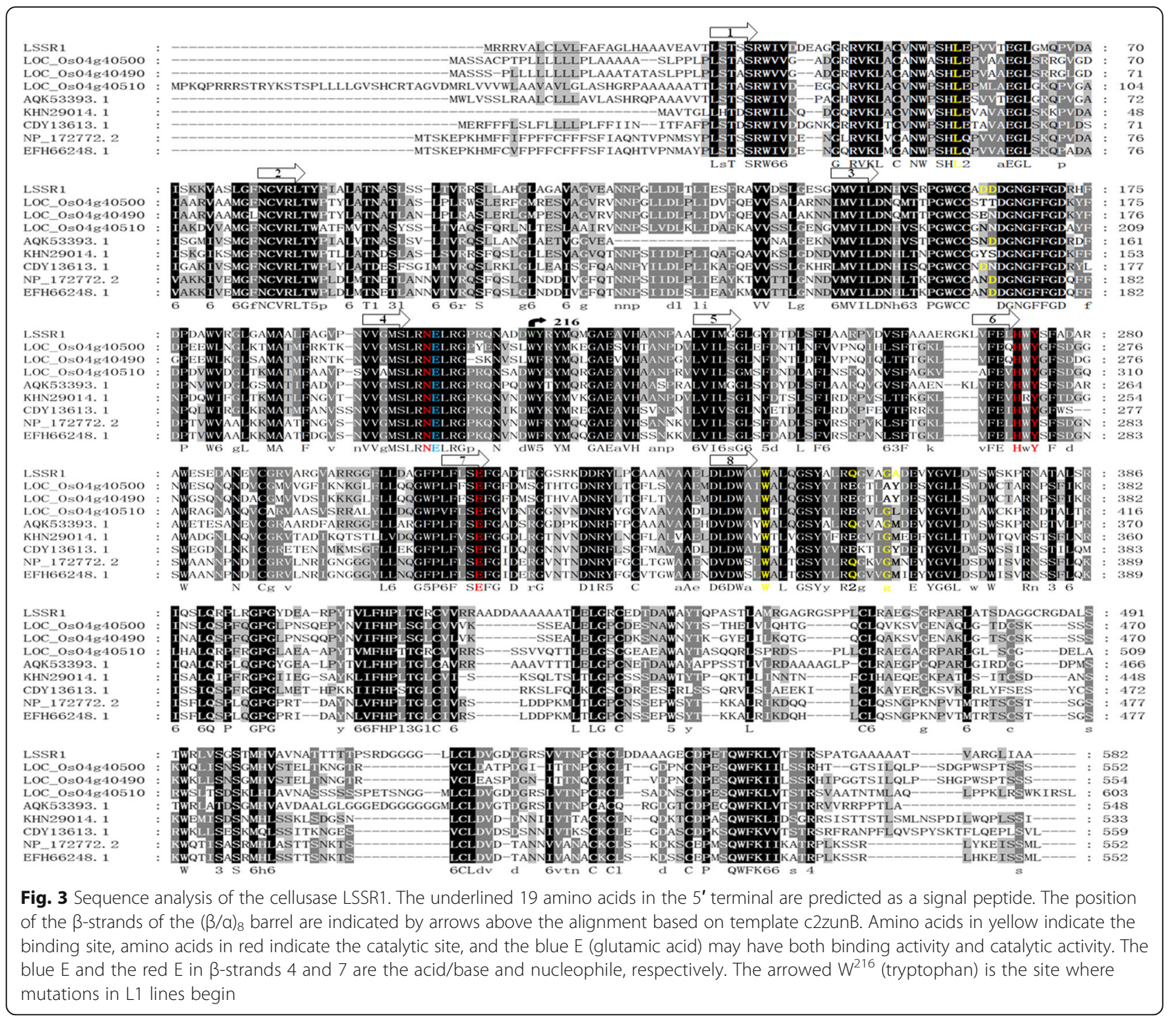

the fluorescence of EGFP alone (Additional file 1: Figure S2). All these results suggest that LSSR1 can be localized to the nucleus and cytoplasm when heterologously over-expressed in $N$. benthamiana.

\section{Issr1 exhibits significant low seed setting rate}

Since no study on the function of LSSR1 or its homologs has been done, a reverse genetic approach was used to examine the physiological role of LSSR1. The CRISPR/ Cas9 system was used to create lssr 1 mutants. Initially, the first target in the middle of the first exon was selected to knock out LSSR1 (Fig. 5a). 5 effective loss-of-function mutants were obtained, namely L1-1 (-1 bp/-3 bp), L1-2 (-4 bp/+), L1-3 ( $-4 \mathrm{bp} /+), \mathrm{L} 1-4$ $(-3 \mathrm{bp} /-)$, and L1-5 (-1 bp/-) (Fig. 5b). As the allelic -3 bp deletion in L1-1 was same as that of mutants L14 , the $-1 \mathrm{bp}$ deletion homozygotes were chosen for seed-setting rate evaluation from the progeny of L1-1.
L1-4 was predicted to produce LSSR1 peptides without the conserved $\mathrm{W}^{216}$ (tryptophan), while the remaining 4 mutants were assumed to generate truncated polypeptides as a result of non-triple-base deletion (Additional file 1: Figure S3). Significantly decreased seed-setting rates were observed in the homozygous progeny of LSSR1 knockout lines when compared with that of the wild type (WT) (Fig. 6a, 7b). The seed setting rate of the WT was $82.43 \%$, while only $42.94 \%, 33.56 \%, 37.02 \%, 24.68 \%$, and $20.16 \%$ plump seeds were harvested in the homozygous $\mathrm{T}_{3}$ lines of L1-1 L1-5, respectively (Fig. 6a).

To confirm that the defective phenotype in the lssr1 mutant lines resulted from the mutation in LSSR1, co-segregation analysis between the phenotype with the genotype in the segregated $\mathrm{T}_{2}$ population of L1-2 and L1-3 was conducted. Complete co-segregation was observed between the defected phenotype and the mutated genotype in L1-2 $(n=50)$ and L1-3 $(n=50)$ (Fig. 6c). 

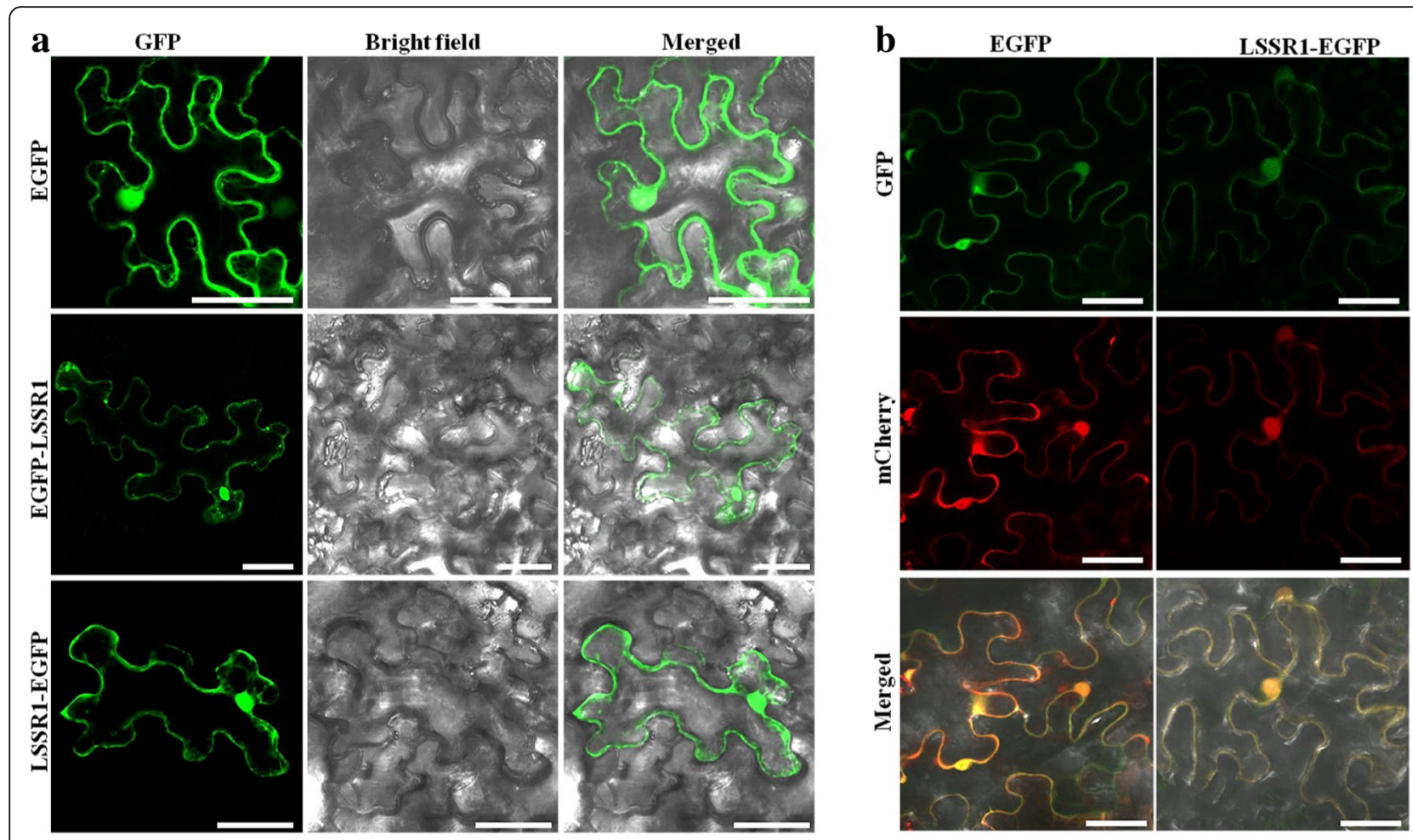

mCherry. Bars $=50 \mu \mathrm{m}$

Additionally, the plants in the segregated $\mathrm{T}_{2}$ population of L1-2 $\left(\chi^{2}=1.24, P=0.27\right)$ and L1-3 $\left(\chi^{2}=0.26, P=0.61\right)$ segregated plants of normal and low seed setting rate in a 3:1 ratio (Additional file 1: Table S2), suggesting that the low seed setting rate of lssr 1 lines is caused by a single recessive mutation in the sporophyte. To reconfirm the causality between the low seed setting rate with the mutated LSSR1, two additional CRISPR targets for LSSR1 were selected to create new lssr1 mutants (Fig. 5a). Four discernible frameshift mutants, namely L2 $-1(-10 \mathrm{bp} /+1$

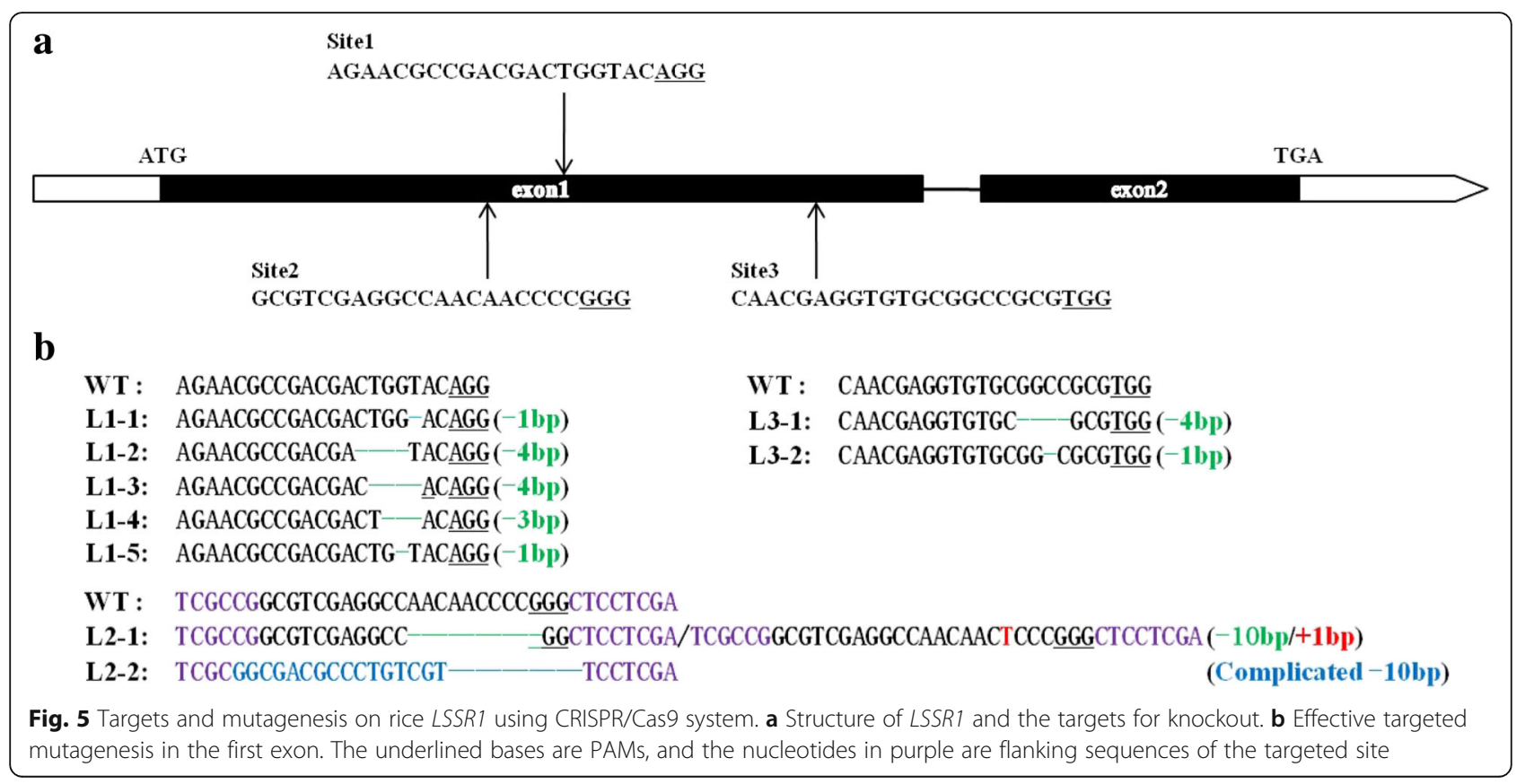



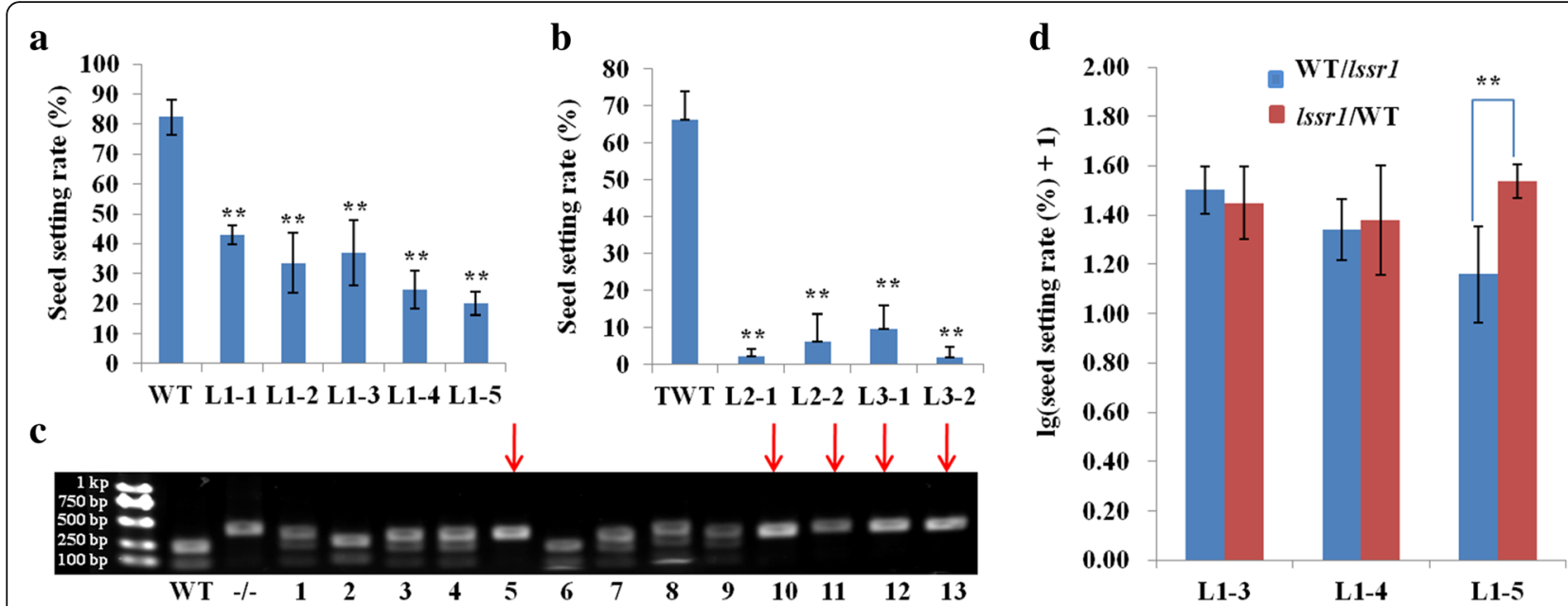

Fig. $\mathbf{6}$ Issr1 exhibits significant low seed setting rate. a Seed setting rate of Issr1 lines with mutation in the first targeted site. Data are means \pm SD from $\geq 6$ individuals with 5 main panicles per plant. b Seed setting rate of the subsequent Issr 1 knockout individuals targeted in site 2 and site3. TWT indicates the transgenic plant without mutation in LSSR1. Data are means + SD with 5 main panicles. c Partial image of the co-segregation analysis in segregated $T_{2}$ populations. The genotypes were assessed by CAPS primer $38260 \mathrm{~s} 1-\mathrm{F} / \mathrm{R}$ and restriction enzyme BseNI. $-/-$ is one of a $T_{2}$ individual from homozygous Issr line L1-5, and red arrows indicate $T_{2}$ individuals with a low seed setting rate from heterozygous /ssr $1 /+$ line L13 when compared with the WT. d Reciprocal crosses between the WT and Issr1 mutant lines. Data are means \pm SD of lg(seed setting rate(\%) +1 ) (for normalization) with $\geq 8$ panicles. "*** indicates a significant difference at $P<0.01$ by the Student's t-test between the WT and L1 lines in $\mathbf{a}$, between TWT and L2 or L3 individuals in $\mathbf{b}$, and between the counterpart combinations in $\mathbf{d}$

bp), L2-2 (Complicated -10 bp/-), L3-1 (-4 bp/-), and L3-2 (-1 bp/-) were obtained from site 2 and site 3 , respectively (Fig. 5b). The seed setting rates of these mutants in $\mathrm{T}_{0}(1.96 \% \sim 9.59 \%)$ were significantly lower than that of the transgenic plant without mutation in LSSR1 (TWT, 66.25\%) (Fig. 6b). These results indicate that the low seed-setting-rate phenotype of lssr 1 lines is exactly caused by the mutated LSSR1.

Reciprocal crosses between the WT and lssr1 lines were conducted to determine whether it was the impaired stamens or pistils in the lssr 1 lines that resulted in a low seed setting rate. L1-3 (4 bp deletion), L1-4 (3 bp deletion), and L1-5 (1 bp deletion), the three lines with typical mutations, were chosen for reciprocal cross assay. When homozygous L1-5 plants were used as the pollen donors, the seed setting rate was significantly lower than that of the counterpart combination (Fig. $6 \mathrm{~d})$. This suggests that the male gamete may be responsible for reduced reproductive success in lssr 1 mutants. No difference in the other two pairs of combinations was found (Fig. 6d), which may be caused by excess pollination of the partially defective $l s s r 1$ pollens.

The number of pollen tubes that reached the Issr 1 ovules is significantly compromised

Because of the similar phenotype in all the independent and stable transgenic lines, L1-3, L1-4, and L1-5 homozygous $\mathrm{T}_{3}$ lines were used in subsequent experiments. lssr 1 lines showed normal vegetative and floret organs in their morphology (Fig. 7a, c). Also, there was no obvious difference in the morphology of the stamens and pistils between lssrl lines and the WT (Fig. 7d, e). Moreover, the pollen $\mathrm{I}_{2}-\mathrm{KI}$ staining appeared normal in the lssr 1 mutant lines when compared to that of the WT (Fig. 7f). Therefore, we supposed that the abortion in lssr1 lines may occur in the process of fertilization, or embryo and endosperm development.

To monitor the fertilization process in lssr 1 lines and the WT, the germination of pollen grains and pollen tube growth were compared in vivo. At 5-30 min after flowering (MAF), abundant pollen grains germinated on the WT stigmas, whereas few or zero germinated pollens were detected on most of the lssr 1 stigmas (Fig. 8a-e). On some of the lssr 1 stigmas, pollen-tube-like dot lines were observed, while on some of the lssr 1 stigmas, no signal could be found (Fig. 8d, e). At 90-120 MAF, about $97.85 \%$ of the WT pistils had pollen tube tips arriving at the basal ovules, but the number of $l s s r 1$ ovules with pollen tubes was greatly reduced (L1-3, 40.77\%; L1-4, 38.15\%; L1-5, 50.79\%) (Fig. 81). In the ovules of the WT, abundant pollen tubes could be seen, while in lssr 1 ovules, few or zero pollen tubes were detected (Fig. $8 \mathrm{f}-\mathrm{h}$ ). Furthermore, some lssr 1 pistils had pollen tubes retarded in various positions (Fig. 8i-k). To evaluate whether the landing of pollen grains on the stigmas in lssr 1 lines is normal, pistils just after flowering in the room were collected and examined using the microscope (LEICA DM4 B, Germany). The number of pollen grains on all of the lssr 1 stigmas (Additional file 1: Figure S4) was more than 20, being sufficient to accomplish the fertilization (Sawada 1974). As the pollen exine plays a 

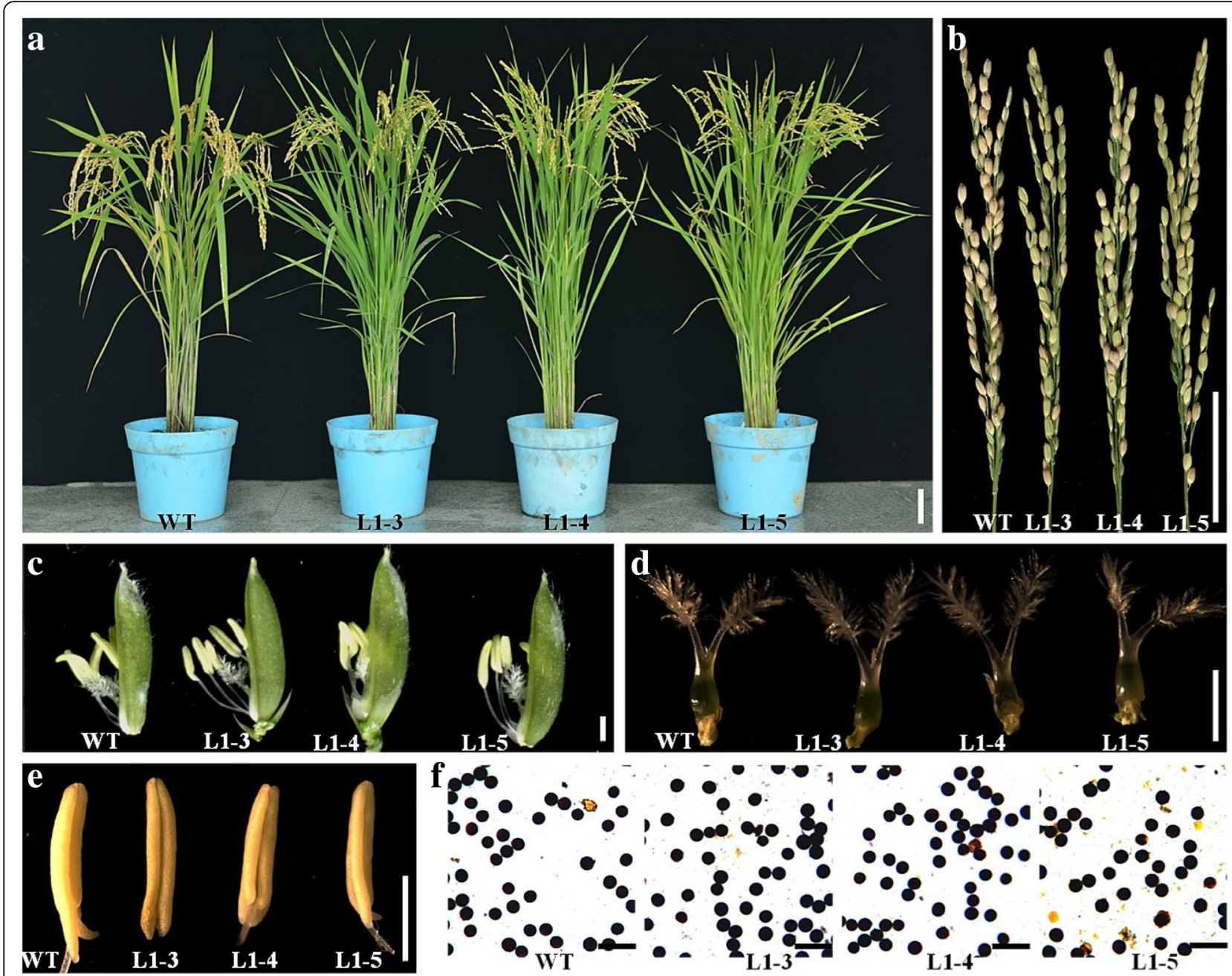

Fig. 7 Phenotypic characterization of Issr1 mutant lines. a Plant morphology of the WT and Issr1 mutants at mature stage. b Mature panicles. c Florets. $\mathbf{d}$ Pistils. e Stamens. $\mathbf{f} \mathrm{I}_{2}-\mathrm{Kl}$ staining of pollen grains. Bars $=5 \mathrm{~cm}$ in $\mathbf{a}$ and $\mathbf{b}, 1 \mathrm{~mm}$ in $\mathbf{c}-\mathbf{e}$, and $100 \mu \mathrm{m}$ in $\mathbf{f}$

critical role in pollen adherence and hydration, scanning electron microscopy (SEM) observation was conducted to observe the morphology of pollen surface in the WT and lssr 1 lines. The morphologic surface of both the mature anther and pollen grains in lssr1 lines showed no difference with that of the WT (Additional file 1: Figure S5). As such, the pollen grain adherence and hydration on the stigma may be normal in $l s s r 1$ lines. These results suggest that the low seed-setting rate of the lssr1 lines may result from abnormal pollen germination, pollen tube penetration, and pollen tube growth in the style.

Conserved amino acid $\mathrm{W}^{216}$ impacts the function of LSSR1 Sequence analysis indicates that $\mathrm{W}^{216}$ (tryptophan) is a conserved amino acid of LSSR1 and its homologs (Fig. 3). The deletion of $\mathrm{W}^{216}$ (tryptophan) on LSSR1 also results in a low seed setting rate in L1-4 (Additional file 1: Figure S3; Fig. 6a, 7b), implying that $\mathrm{W}^{216}$ is a functionally important residue for LSSR1. To explore that if the proper folding of
LSSR1 is affected by this residue, the three-dimensional (3D) structure of the functional dormain of both the WT LSSR1 and L1-4 LSSR1 were predicted using Phyre2 with the same template (Fig. 9a). Althrough the overall structure and catalytic groove on the $\mathrm{W}^{216}$-deleted LSSR1 seem to be similar to that of the WT LSSR1, some parts of the binding surface of the mutated LSSR1 change (Fig. 9b-d). In $\mathrm{W}^{216}$-deleted LSSR1, the extended betas on both sides of $\mathrm{L}^{55}$ (leucine) in the WT LSSR 1 changes to be a coil and a turn, the coiled $\mathrm{D}^{163 / 164}$ (aspartatic acid) along with a adjacent residue on both sides becomes a helix, and the loop structure between the acid/base $\mathrm{E}^{205}$ (glutamic acid) and the deleted $\mathrm{W}^{216}$ looses (Fig. 9e-g). These results suggest that $\mathrm{W}^{216}$ may have a great effect on the spatial structure and proper function of LSSR1.

\section{Discussion}

In this study, we describe a novel cellulase encoding gene LSSR1 in rice. Dysfunction of LSSR1 results in a 


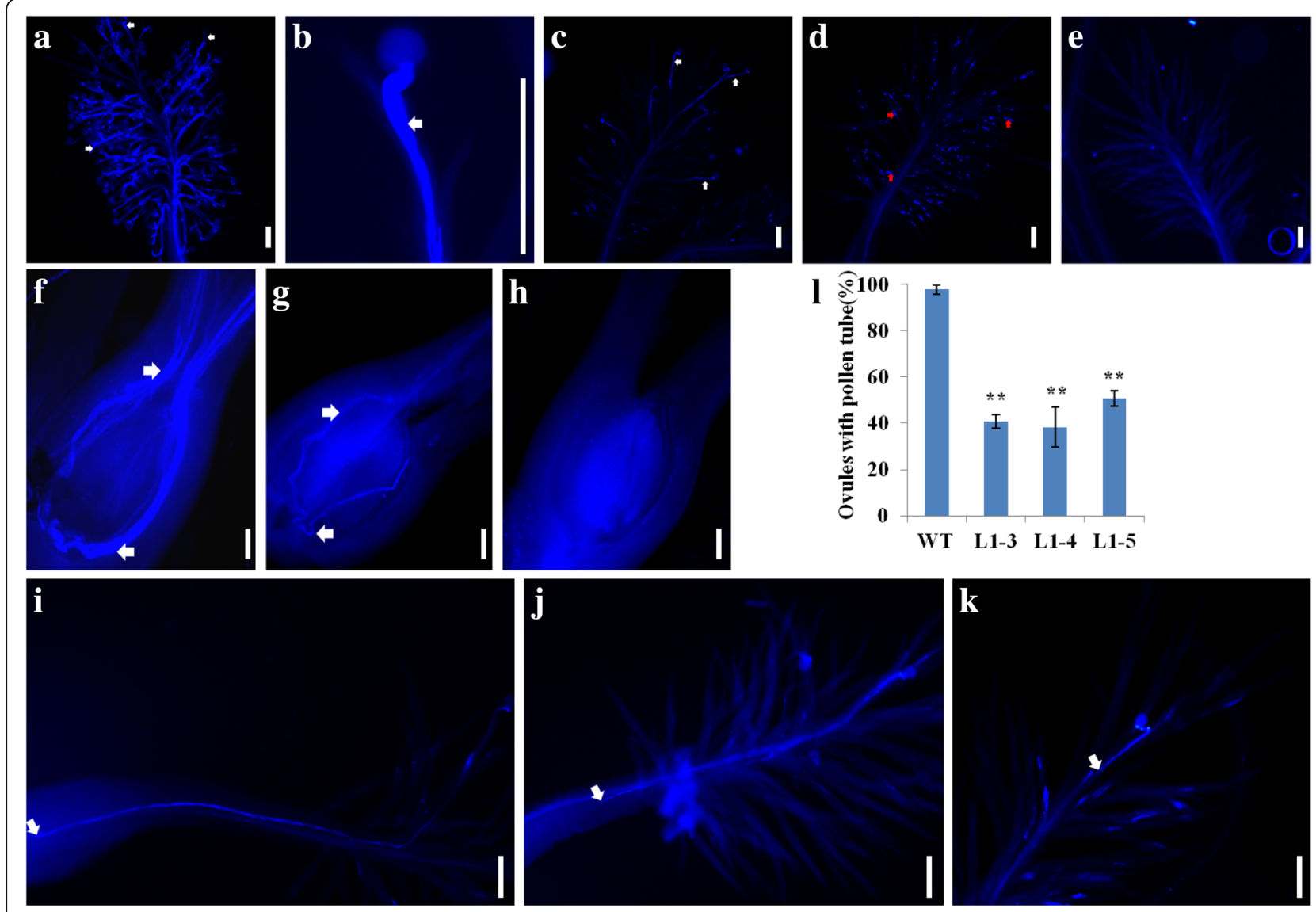

Fig. 8 Pollen tube germination and growth of the WT and Issr1 lines in vivo. a Aniline blue staining of the WT pistils at 5-30 min after flowering (MAF). b Amplified pollen tube of the WT. c-e Little or no germinated pollen grains were detected on the Issr1 stigma at 5-30 MAF. $\mathbf{f}$ Multiple pollen tubes reached the ovules of the WT at 90-120 MAF. $\mathbf{g}$ and $\mathbf{h}$ few or zero pollen tubes reached the ovules of Issr 1 lines at 90-120 MAF. i-k Retardation of pollen tube growth in Issr 1 lines at 90-120 MAF. Bars $=100 \mu \mathrm{m}$. White arrows point to pollen tubes in a-c, $\mathbf{f}$ and $\mathbf{g}$, and pollen tube tips in i-k. Red arrows point to pollen-tube-like dot lines on the stigma. I Quantification of ovaries with pollen tubes observed in the ovule at 90-120 MAF. Data are means \pm SD from 3 replicates with $\geq 23$ pistils per replicate, and "**" indicates a significant difference between the WT and Issri lines at $P<0.01$ by the Student's t-test

low seed setting rate, but the morphology of the vegetative and reproductive organs, as well as the pollen $\mathrm{I}_{2}-\mathrm{KI}$ staining, appears normal in lssr1 mutant lines (Fig. 6a, b; 7). Our cytological studies suggest that it is most likely the blockage of fertilization that leads to the decreased seed setting rate in $l s s r 1$ lines. The majority of $l s s r 1$ pistils had few or no germinated pollen grains adhering to the stigma at 5-30 MAF, but pollen-tube-like dot lines remained on some lssr1 stigmas (Fig. 8a-e). At 90-120 MAF, the number of $l s s r 1$ ovules with pollen tubes was significantly lower than that of the WT (Fig. 81). Additionally, retarded pollen tubes were detected in lssr1 pistils at 90-120 MAF (Fig. 8i-k). As more than 20 pollen grains could be observed on the $l s s r 1$ stigmas just after flowering (Additional file 1: Figure S4), we deduce that the landing of pollens on the stigma in lssrl lines does not account for their abnormal fertilization. In Arabidopsis (Arabidopsis thaliana), the lipophilic molecules in the pollen exine mediate the pollen-stigma adhesion (Zinkl et al. 1999), while the aperture number influences pollen survival against water inflow (Prieu et al. 2016), suggesting that the pollen exine is of great importance for pollen adherence and hydration on the stigma. Nonetheless, the morphology of the pollen epidermal surface of lssr1 lines showed no difference with that of the WT (Additional file 1: Figure S5h1-h4). Therefore, the pollen adherence and hydration on the stigma in lssr 1 lines are probably normal. In conclusion, the arrested fertilization in lssr 1 lines may result from the aborted pollen grain germination, failed pollen tube penetration, and unsuccessful elongation of pollen tubes to the ovules. Additional experiments are needed to validate these results and find out the critical barrier for the successful fertilization in lssr 1 lines.

Any deviation during fertilization in flowering plants could result in sterility. The OsMLO12 protein contains 


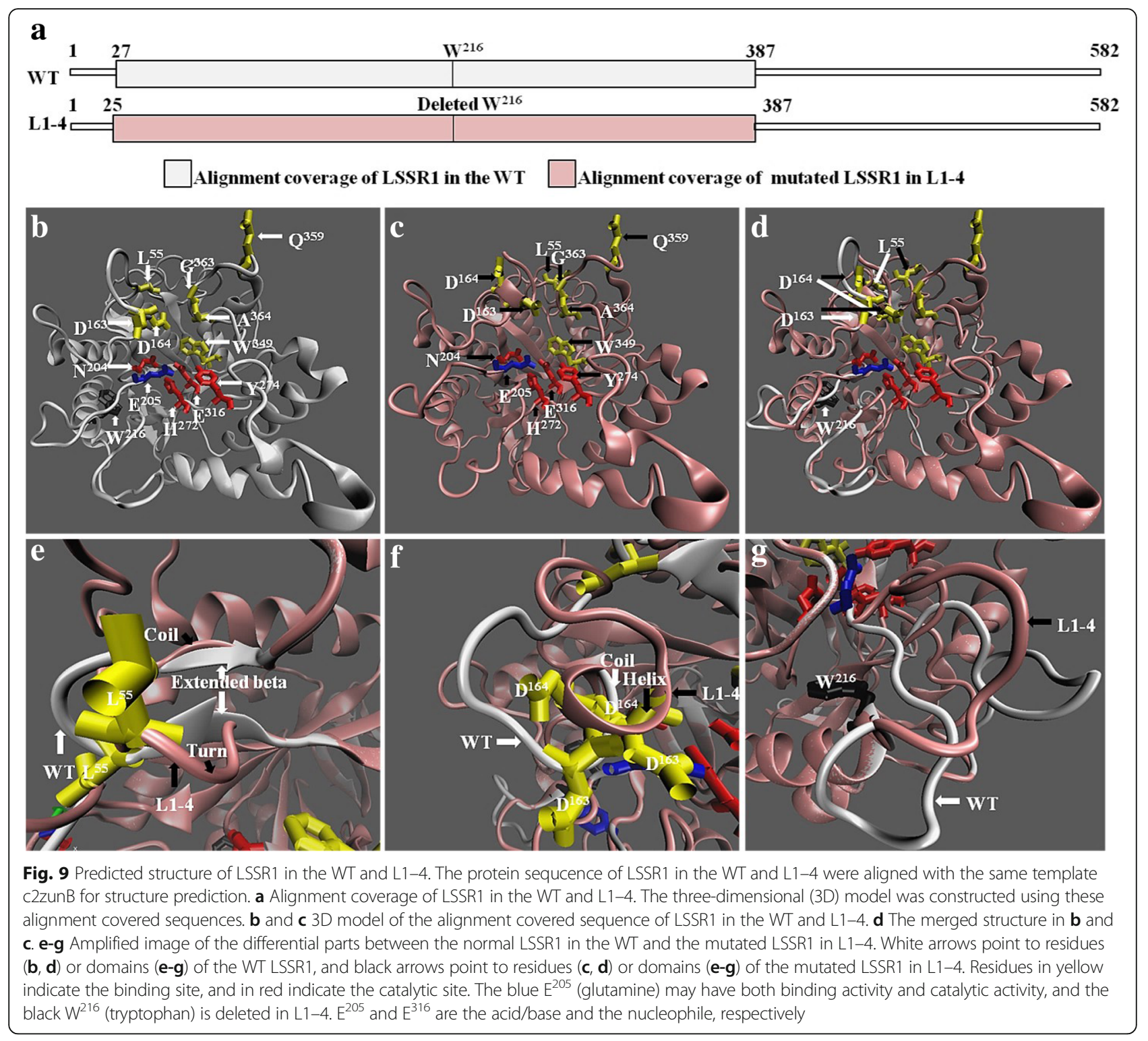

seven transmembrane motifs, which regulates pollen hydration through an interaction with calmodulin in the cytosol (Yi et al. 2014). Osmlo12 pollens matured normally with three nuclei, but could not germinate in vitro or in vivo due to a failure in hydration (Yi et al. 2014). The disruption of rice aspartic protease OsAP65 caused the dysfunction in pollen tube germination and elongation (Huang et al. 2013). AtOFT1, a putative protein O-fucosyltransferase in Arabidopsis, promotes pollen tube penetration through the stigma-style interface (Smith et al. 2018). oft1 pollen tubes could germinate normally, but their ability to penetrate through the stigma during fertilization was largely compromised (Smith et al. 2018). BUPS1/2, as the receptors of signals from the female cells in Arabidopsis, coordinate the pollen tube integrity and sperm release by interacting with RAF4/19 and ANXUR1/2 (Ge et al. 2017). bups1 bups 2 pollen tubes ruptured immediately upon germination (Ge et al. 2017). All of these genes regulate plant fertility through gametophyte and showed segregation distortion in the progeny of heterozygous mutants. However, the segregation ratios in the progeny of lssr 1 heterozygous mutants were in accordance with Mendelian inheritance (Additional file 1: Table S2), indicating that LSSR1 control rice fertility through the sporophyte.

Cellulose, the main load-bearing polysaccharide in all cell walls of higher plants (Mélida et al. 2009), may be the critical barrier for rice fertilization. Previous research has suggested that the pollen tube must penetrate the cuticle and cell wall on the stigma to create an entry for further penetration (Jiang 2005). In this process, wall hydrolases, including cellulases, either derived from the 
tapetum cells of developing anthers or rapidly synthesized by pollens, play a significant role. ZmXYN1 is an endoxylanase in maize, whose mRNA is located in the tapetum cells enclosing the developing pollen in anthers, while the mature $35-\mathrm{kDa}$ endoxylanase is the predominant protein in the maize pollen coat (Wu et al. 2002). Xyl-less (Transgenic lines containing little or no xylanase in the pollen coat) pollen tubes germinated and elongated comparably with that of the WT pollen tubes, but did not penetrate into the silk as efficiently as pollen tubes of the WT (Suen and Huang 2006). Another major protein in the maize pollen coat is $Z m$ GLA3, a $70-\mathrm{kDa}$ $\beta$-glucanase, which is also derived from the tapetum in developing anthers and may hydrolyze the stigma wall for pollen tube entry (Suen et al. 2003). LSSR1 is predicted as a cellulase. The qRT-PCR analysis showed that LSSR1 is predominantly expressed in anthers during the developmental stage of microsporogenesis, including the premeiosis, meiosis, and single-cell pollen stages (Fig. 1b-f). Like EGFP or mCherry alone control, the encoded protein of LSSR1 can be localized to the nucleus and cytoplasm in N. benthamiana (Fig. 4; Additional file 1: Figure S2), which is inconsistent with the putative secretion of LSSR1. Therefore, $m$ Cherry-fused complementation lines will be created to confirm the localization of LSSR1 in our future study. We suppose that LSSR1 is synthesized in the tapetum cells and transported to the developing microspores, and functions during rice fertilization. However, we have not yet obtained the purified functional protein to evaluate its catalytic activity and substrates.

When comparing with template c2zunB, 5 catalytic sites and 8 binding sites of LSSR1, including the acid/ base and the nucleophile, were predicted, respectively (Fig. 3). In addition, some other amino acid residues conserved in LSSR1 and its homologs were marked with black background (Fig. 3). A deletion of the conserved $\mathrm{W}^{216}$ (tryptophan) in L1-4 leads to the defective phenotype, just as other garbled and truncated mutations do (Additional file 1: Figure S3; Fig. 6a, 7b). This implies that $\mathrm{W}^{216}$ is a functionally important residue for LSSR1. Structure analysis showed some difference on the binding surface between the WT LSSR1 and $\mathrm{W}^{216}$-deleted LSSR1 (Fig. 9d-g). These results indicate that the proper folding of LSSR1 and its normal function is largely impacted by the conserved $\mathrm{W}^{216}$. Furthermore, elucidating the function of the putative active sites in LSSR1 will also be the subject of future studies.

Morever, the partial defect of seed setting in $l s s r 1$ lines indicates that other cellulases or hydrolases with cellulase activity in or on the surface of pollen grains may function during rice fertilization. There are 25 rice GH5 proteins in the CAZy database, most of which are putative mannosidases. The $\beta$-glucosidase GH5BG is one of the GH5 proteins in rice, which may be a regulator in response to stress in rice seedlings (Opassiri et al. 2007). No functional analysis has been made in other rice GH5 proteins. LSSR1 and its three paralogs (LOC_Os04g40490, LOC_Os04g40500 and LOC_Os04g40510) are predicted to be cellulases (Fig. 2b), but they may have various expression patterns according to the data from the Rice eFP Browser. More studies are required to clarify whether the paralogs of LRR1 or other hydrolases coordinate with LRR1 to hydrolyze celluloses in the papillar cell walls.

Overall, this study provides strong evidence to suggest that the novel gene LSSR1 plays a critical role in controlling the seed setting rate of rice. It most likely does this by controlling the fertilization process. No cellulase gene had ever been linked to rice seed setting rate previously. The discovery of a low seed setting rate in lssr 1 mutants provides a unique opportunity to use cell biological techniques (such as in vitro pollen tube growth assay and semi-in-vivo assay) and robust genetics to investigate how GH5 cellulases function during plant fertilization, as well as in rice seed setting.

\section{Additional file}

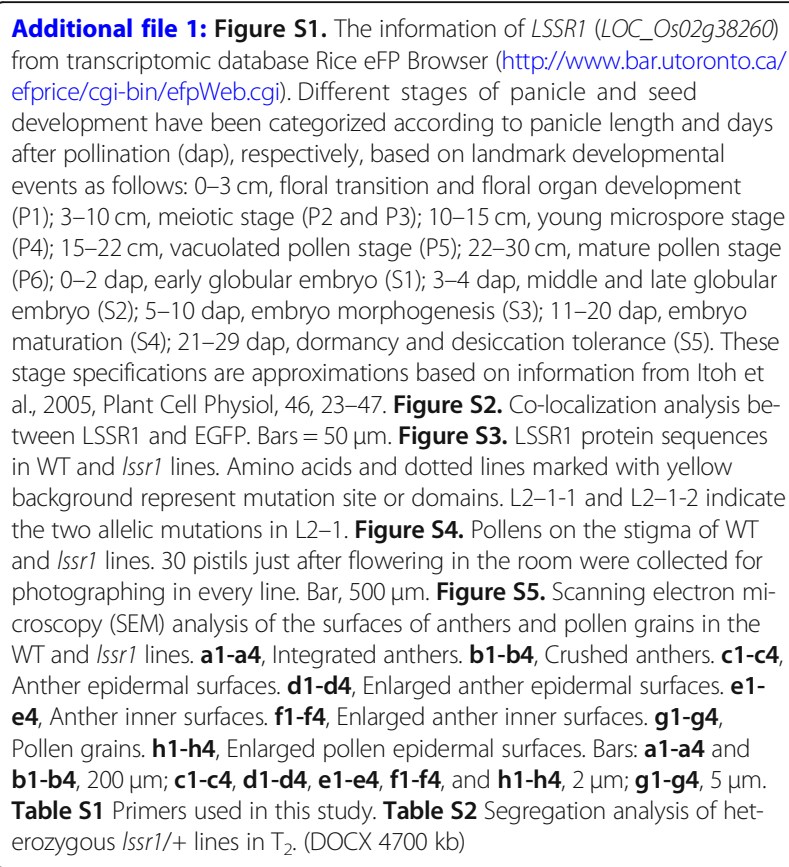

\section{Abbreviations}

3D: Three-dimensional; $\mathrm{Ca}^{2+}$ cyt: Cytoplasmic calcium concentration; CAPS: Cleaved polymorphic amplified sequence; ECM: Extracellular matrix; FAA: Formalin-aceto-alcohol; GH: Glycosyl hydrolase; MAF: Minutes after flowering; SEM: Scanning electron microscopy; STT: Style transmission tissue; TT: Transmitting tract

\section{Acknowledgments}

We thank Bio-ultrastructure analysis Lab. of Analysis center of Agrobiology and environmental sciences, Zhejiang Univ. for scanning electron microscopy (SEM) observation. 


\section{Funding}

This work was supported by grants from the National Natural Science Foundation of China (\#31521064), the National Key Transform Program (\#2016ZX08001-002), the Chinese Academy of Agricultural Sciences Innovation Project (CAAS-ASTIP2013-CNRRI), and the State Key Laboratory of Rice Biology (2017ZZKT10202).

\section{Availability of data and materials}

All data generated or analysed during this study are included in this article and its additional file. And all materials are available in our lab.

\section{Authors' contributions}

$X X, P Z, Y Z, S C$ and $L C$ designed the research. XX performed the research, analyzed the data, and wrote the manuscript. PZ, PY, LS, ZY, RMK, AA, and WW provided assistance in data collection and manuscript revision. All authors have read the manuscript and approved to submit it to your journal.

\section{Ethics approval and consent to participate}

Not applicable.

\section{Consent for publication}

Not applicable.

\section{Competing interests}

The authors declare that they have no competing interests.

\section{Publisher's Note}

Springer Nature remains neutral with regard to jurisdictional claims in published maps and institutional affiliations.

\section{Author details}

${ }^{1}$ Key Laboratory for Zhejiang Super Rice Research and State Key Laboratory of Rice Biology, China National Rice Research Institute, Hangzhou 310006, China. ${ }^{2}$ National Key Laboratory of Crop Genetic Improvement, Huazhong Agricultural University, Wuhan 430070, China.

\section{Received: 12 December 2018 Accepted: 25 March 2019}

\section{Published online: 09 May 2019}

\section{References}

Ashikari M, Sakakibara H, Lin S, Yamamoto T, Takashi T, Nishimura A, Angeles ER, Qian Q, Kitano H, Matsuoka M (2005) Cytokinin oxidase regulates rice grain production. Science 309:741-745.

Bosch M, Hepler PK (2005) Pectin methylesterases and pectin dynamics in pollen tubes. Plant Cell 17:3219-3226.

Carpita NC, Gibeaut DM (1993) Structural models of primary cell walls in flowering plants: consistency of molecular structure with the physical properties of the walls during growth. Plant J 3:1-30.

Dan Z, Liu P, Huang W, Zhou W, Yao G, Hu J, Zhu R, Lu B, Zhu Y (2014) Balance between a higher degree of heterosis and increased reproductive isolation: a strategic design for breeding inter-subspecific hybrid rice. PLoS One 9: e93122.

Deveshwar P, Bovill WD, Sharma R, Able JA, Kapoor S (2011) Analysis of anther transcriptomes to identify genes contributing to meiosis and male gametophyte development in rice. BMC Plant Biol 11:78

Duan P, Xu J, Zeng D, Zhang B, Geng M, Zhang G, Huang K, Huang L, Xu R, Ge S, Qian Q, Li Y (2017) Natural variation in the promoter of GSE5 contributes to grain size diversity in rice. Mol Plant 10:685-694.

Ge Z, Bergonci T, Zhao Y, Zou Y, Du S, Liu M, Luo X, Ruan H, GarciaValencia LE, Zhong S, Hou S, Huang Q, Lai L, Moura DS, Gu H, Dong J, Wu H, Dresselhaus T, Xiao J, Cheung AY, Qu L (2017) Arabidopsis pollen tube integrity and sperm release are regulated by RALF-mediated signaling. Science 358:1596-1600.

Henrissat B, Davies G (1997) Structural and sequence-based classification of glycoside hydrolases. Curr Opin Struc Bio 7:637-644.

Huang J, Zhao X, Cheng K, Jiang Y, Ouyang Y, Xu C, Li X, Xiao J, Zhang Q (2013) OsAP65, a rice aspartic protease, is essential for male fertility and plays a role in pollen germination and pollen tube growth. J Exp Bot 64:3351-3360.

Huang X, Peng X, Sun MX (2017) OsGCD1 is essential for rice fertility and required for embryo dorsal-ventral pattern formation and endosperm development. New Phytol 215:1039-1058.
Huo X, Wu S, Zhu Z, Liu F, Fu Y, Cai H, Sun X, Gu P, Xie D, Tan L, Sun C (2017) NOG1 increases grain production in rice. Nat Commun 8:1497.

Ishimaru K, Hirotsu N, Madoka Y, Murakami N, Hara N, Onodera H, Kashiwagi T, Ujiie K, Shimizu B, Onishi A, Miyagawa H, Katoh E (2013) Loss of function of the IAA-glucose hydrolase gene TGW6 enhances rice grain weight and increases yield. Nat Genet 45:707-711.

Jenkins J, Leggio LL, Harris G, Pickersgill R (1995) $\beta$-Glucosidase, $\beta$-galactosidase, family a cellulases, family $F$ xylanases and two barley glycanases form a superfamily of enzymes with 8-fold $\beta / \alpha$ architecture and with two conserved glutamates near the carboxy-terminal ends of $\beta$-strands four and seven. FEBS Lett 362:281-285.

Jiang L (2005) VANGUARD1 encodes a pectin methylesterase that enhances pollen tube growth in the Arabidopsis style and transmitting tract. Plant Cell 17:584-596.

Kelley LA, Mezulis S, Yates CM, Wass MN, Sternberg MJE (2015) The Phyre2 web portal for protein modeling, prediction and analysis. Nat Protoc 10:845-858.

Kim H, Ishikawa K (2011) Functional analysis of hyperthermophilic endocellulase from Pyrococcus horikoshii by crystallographic snapshots. Biochem J 437:223-230.

Leydon AR, Tsukamoto T, Dunatunga D, Qin Y, Johnson MA, Palanivelu R (2015) Pollen tube discharge completes the process of synergid degeneration that is initiated by pollen tube-synergid interaction in Arabidopsis. Plant Physiol 169:485-496

Li D, Huang Z, Song S, Xin Y, Mao D, Lv Q, Zhou M, Tian D, Tang M, Wu Q, Liu X, Chen T, Song X, Fu X, Zhao B, Liang C, Li A, Liu G, Li S, Hu S, Cao X, Yu J, Yuan L, Chen C, Zhu L (2016) Integrated analysis of phenome, genome, and transcriptome of hybrid rice uncovered multiple heterosis-related loci for yield increase. Proc Natl Acad Sci U S A 113:E6026-E6035.

Li S, Li W, Huang B, Cao X, Zhou X, Ye S, Li C, Gao F, Zou T, Xie K, Ren Y, Ai P, Tang Y, Li X, Deng Q, Wang S, Zheng A, Zhu J, Liu H, Wang L, Li P (2013) Natural variation in PTB1 regulates rice seed setting rate by controlling pollen tube growth. Nat Commun 4:2793.

Li X, Qian Q, Fu Z, Wang Y, Xiong G, Zeng D, Wang X, Liu X, Teng S, Hiroshi F, Yuan M, Luo D, Han B, Li J (2003) Control of tillering in rice. Nature 422:618-621.

Li Y, Fan C, Xing Y, Jiang Y, Luo L, Sun L, Shao D, Xu C, Li X, Xiao J, He Y, Zhang $Q$ (2011) Natural variation in GS5 plays an important role in regulating grain size and yield in rice. Nat Genet 43:1266-1269.

Liu Q, Ning Y, Zhang Y, Yu N, Zhao C, Zhan X, Wu W, Chen D, Wei X, Wang G, Cheng S, Cao L (2017) OsCUL3a negatively regulates cell death and immunity by degrading OsNPR1 in rice. Plant Cell 29:345-359.

Livak KJ, Schmittgen TD (2001) Analysis of relative gene expression data using real-time quantitative PCR and the 2- ${ }^{\Delta \Delta C T}$ method. Methods 25:402-408.

Márton M, Dresselhaus T (2010) Female gametophyte-controlled pollen tube guidance. Biochem Soc Trans 38:627-630.

Mélida H, García-Angulo P, Alonso-Simón A, Encina A, Álvarez J, Acebes JL (2009) Novel type II cell wall architecture in dichlobenil-habituated maize calluses. Planta 229:617-631.

Miao J, Guo D, Zhang J, Huang Q, Qin G, Zhang X, Wan J, Gu H, Qu L (2013) Targeted mutagenesis in rice using CRISPR-Cas system. Cell Res 23:1233-1236

Murray MG, Thompson WF (1980) Rapid isolation of high molecular weight plant DNA. Nucleic Acids Res 8:4321-4326.

Opassiri R, Pomthong B, Akiyama T, Nakphaichit M, Onkoksoong T, Ketudat Cairns M, Ketudat Cairns JR (2007) A stress-induced rice (Oryza sativa L.) $\beta$ glucosidase represents a new subfamily of glycosyl hydrolase family 5 containing a fascin-like domain. Biochem J 408:241-249.

Palanivelu R, Johnson MA (2010) Functional genomics of pollen tube-pistil interactions in Arabidopsis. Biochem Soc Trans 38:593-597.

Prieu C, Matamoro-Vidal A, Raquin C, Dobritsa A, Mercier R, Gouyon P, Albert B (2016) Aperture number influences pollen survival in Arabidopsis mutants. Am J Bot 103:452-459.

Sandaklie-Nikolova L, Palanivelu R, King EJ, Copenhaver GP, Drews GN (2007) Synergid cell death in Arabidopsis is triggered following direct interaction with the pollen tube. Plant Physiol 144:1753-1762.

Sawada S (1974) Minimal number of dehiscent anthers and pollen grains on the stigma required for fertilization in rice plants. Res Bull Obihiro Univ 9:165-171.

Seck PA, Diagne A, Mohanty S, Wopereis MCS (2012) Crops that feed the world 7: Rice. Food Secur 4:7-24.

Smith DK, Jones DM, Lau JBR, Cruz ER, Brown E, Harper JF, Wallace IS (2018) A putative protein O-fucosyltransferase facilitates pollen tube penetration through the stigma-style interface. Plant Physiol 176:2804-2818. 
Suen DF, Huang AHC (2006) Maize pollen coat xylanase facilitates pollen tube penetration into silk during sexual reproduction. J Biol Chem 282:625-636.

Suen DF, Wu SSH, Chang HC, Dhugga KS, Huang AHC (2003) Cell wall reactive proteins in the coat and wall of maize pollen. J Biol Chem 278:43672-43681.

Takeda T, Suwa Y, Suzuki M, Kitano H, Ueguchi-Tanaka M, Ashikari M, Matsuoka M, Ueguchi C (2003) The OsTB1 gene negatively regulates lateral branching in rice. Plant J 33:513-520

Wang YP, Tang SQ, Chen HZ, Wu ZF, Zhang H, Duan EC, Shi QH, Wu ZM (2017) Identification and molecular mapping of indica high-tillering dwarf mutant htd4, a mild phenotype allelic mutant of D14 in rice (Oryza sativa L.). Plant Biol 19:851-858.

Wass MN, Kelley LA, Sternberg MJE (2010) 3DLigandSite: predicting ligandbinding sites using similar structures. Nucleic Acids Res 38:W469-W473.

Wu SSH, Suen DF, Chang HC, Huang AHC (2002) Maize tapetum xylanase is synthesized as a precursor, processed and activated by a serine protease, and deposited on the pollen. J Biol Chem 277:49055-49064.

Wu Y, Wang Y, Mi X, Shan J, Li X, Xu J, Lin H (2016) The QTL GNP1 encodes GA20ox1, which increases grain number and yield by increasing cytokinin activity in rice panicle meristems. PLoS Genet 12:e1006386.

Xu C, Wang Y, Yu Y, Duan J, Liao Z, Xiong G, Meng X, Liu G, Qian Q, Li J (2012) Degradation of MONOCULM 1 by APC/CTAD1 regulates rice tillering. Nat Commun 3:750.

Yi J, An S, An G (2014) OsMLO12, encoding seven transmembrane proteins, is involved with pollen hydration in rice. Plant Reprod 27:169-180.

Zhang C, Li G, Chen T, Feng B, Fu W, Yan J, Islam MR, Jin Q, Tao L, Fu G (2018) Heat stress induces spikelet sterility in rice at anthesis through inhibition of pollen tube elongation interfering with auxin homeostasis in pollinated pistils. Rice 11:14

Zhang P, Zhang Y, Sun L, Sinumporn S, Yang Z, Sun B, Xuan D, Li Z, Yu P, Wu W, Wang K, Cao L, Cheng S (2017) The rice AAA-ATPase OsFIGNL1 is essential for male meiosis. Front Plant Sci 8:1639.

Zhou S, Wang Y, Li W, Zhao Z, Ren Y, Wang Y, Gu S, Lin Q, Wang D, Jiang L, Su N, Zhang X, Liu L, Cheng Z, Lei C, Wang J, Guo X, Wu F, lkehashi H, Wang H, Wan J (2011) Pollen Semi-Sterility1 encodes a kinesin-1-like protein important for male meiosis, anther dehiscence, and fertility in rice. Plant Cell 23:111-129.

Zhu S, Lin Q, Jiang L, Wu C, Wang H, Wan J (2017) OsCNGC13 promotes seedsetting rate by facilitating pollen tube growth in stylar tissues. PLoS Genet 13:e1006906.

Zinkl GM, Zwiebel Bl, Grier DG, Preuss D (1999) Pollen-stigma adhesion in Arabidopsis: a species-specific interaction mediated by lipophilic molecules in the pollen exine. Development 126:5431.

Yadegari R, Drews GN (2004) Female gametophyte development. Plant Cell 16: S133-S141.

\section{Submit your manuscript to a SpringerOpen ${ }^{\circ}$ journal and benefit from:}

- Convenient online submission

- Rigorous peer review

- Open access: articles freely available online

- High visibility within the field

- Retaining the copyright to your article

Submit your next manuscript at $\boldsymbol{\nabla}$ springeropen.com 